\title{
Fattori metabolici nella risposta renale all'infusione di amino acidi
}

\author{
A. Claris-Appiani ${ }^{1}$, G. Ardissino ${ }^{1}$, A.S. Tirelli ${ }^{2}$, V. Dacco'1, S. Testa ${ }^{1}$, C. Corbetta ${ }^{2}$, \\ L. Guidi ${ }^{2}$, E. Moretto ${ }^{3}$, B.M. Assael ${ }^{1}$, F. Sereni ${ }^{1}$ \\ ${ }^{1}$ Clinica Pediatrica II, Università di Milano, \\ ${ }^{2}$ Laboratorio e ${ }^{3}$ Farmacia, Istituti Clinici di Perfezionamento - Milano
}

nche se l'incremento dell'emodinamica renale durante infusione di amino acidi (AA) è un fenomeno fisiologico ben definito (1), non è stato ancora chiarito quale sia l'esatto meccanismo alla base di tale risposta. Premen (2) ha dimostrato che l'infusione diretta di AA nell'arteria renale non provoca iperfiltrazione, suggerendo quindi l'esistenza di un passaggio intermedio necessario affinché si verifichi la risposta renale. Numerosi indizi fanno ritenere che tale meccanismo intermedio possa realizzarsi a livello splancnico. Gli AA a catena ramificata, che sono scarsamente metabolizzati a tale livello, stimolano l'emodinamica renale in minor misura rispetto a quelli che vengono transaminati e deaminati dal fegato (serina, prolina, alanina, acidi bicarbossilici e AA gluconeogenetici in generale) (2,3-5). Inoltre il glucagone è stato ripetutamente citato come elemento chiave nel meccanismo di iperfiltrazione renale indotta dagli AA (3,69). In ogni caso, infusioni di glucagone nei ratti non provocano alcun aumento del FG se non quando somministrato in dosi tali da raggiungere i livelli osservati nel circolo portale dopo un pasto proteico (10), oppure se associati a cAMP e urea (11). Alcuni Autori $(1,12)$ hanno ipotizzato che, oltre ai fattori intermedi, sia necessario, come ultimo passaggio della stimolazione dell'emodinamica glomerulare, un meccanismo intrarenale che probabilmente coinvolge il trasporto del $\mathrm{Na}$ e il feedback tubulo glomerulare. In ogni caso non sono state ancora identificate correlazioni tra un possibile meccanismo renale e il metabolismo splancnico degli AA (o fattori ad esso associati).

In questo studio, per stabilire l'eventuale ruolo svolto dal metabolismo degli AA nel determinare le modificazioni dell'emodinamica renale da essi indotte, 7 soggetti volontari sani sono stati sottoposti ad infusione di 2 soluzioni di AA con differente metabolismo. La prima soluzione conteneva AA essenziali (EAA), che sfuggono al metabolismo splancnico; l'altra, una miscela di AA (MIX-AA) che includeva 5 AA non essenziali, elettivamente metabolizzati a livello epatico (serina, prolina, arginina, alanina e glicina) e con un'elevata capacità stimolante il glucagone. Quindi è stata analizzata la correlazione tra la risposta splancnica (produzione di urea, livelli plasmatici di glucagone e insulina) e quella renale a tali infusioni.

\section{Metodi}

Sono stati selezionati per lo studio 7 soggetti maschi sani con età compresa tra 21 e 25 anni. Essi avevano un' anamnesi patologica remota negativa, non avevano segni clinici o laboratoristici della presenza di patologie a carico dell'apparato renale, endocrino, cardiovascolare o altro apparato. Nessuno di essi era in terapia con alcun farmaco e non ne aveva assunti nella settimana precedente lo studio. Ogni soggetto è stato sottoposto a 2 protocolli sperimentali, fungendo quindi da controllo di se stesso: tra l'esecuzione dei due è intercorso un intervallo di almeno 2 settimane. È stata eseguita una trascrizione dietetica e sono state determinate l'escrezione urinaria di $\mathrm{Na}$ e urea nei 3 giorni precedenti entrambe le infusioni, allo scopo di quantificare l'apporto dietetico delle due sostanze; non sono state osservate differenze con l'apporto stimato di $\mathrm{Na}$ e proteine in entrambi i casi. Tutti gli esami sono stati eseguiti al mattino a digiuno. Ogni soggetto ha fornito il suo consenso informato e lo studio è stato approvato dal Comitato etico dell'Ospedale. 


\section{Protocollo sperimentale}

Il test iniziò alle 8.00 a.m. con l'infusione, attraverso vena cubitale, della prima dose di polifruttosano S (Inutest $25 \%$, Laevosan Gesellschaft, Linz, Austria) e di acido p-aminoippurico (PAH 5\%, Monico, Italia), alla velocità di $1.1 \mathrm{ml} / \mathrm{min}$ per $1.5 \mathrm{mi}$ nuti. A tale bolo iniziale seguì un'infusione continua con velocità determinata sulla base del peso corporeo di ogni soggetto e con lo scopo di raggiungere e mantenere un valore plasmatico di $20 \mathrm{mg} / \mathrm{dl}$ per l'Inutest e di $2 \mathrm{mg} / \mathrm{dl}$ per il PAH (velocità media di $0.11 \mathrm{ml} / \mathrm{min}$ per Inutest al $25 \%$ e PAH al 13\%). La quantità media di liquidi infusi nelle 4 ore fu compresa tra i 70 e gli $80 \mathrm{ml}$. Entro 20 minuti dall'inizio del test e per tutta la durata dello stesso, fu somministrata acqua per os $(20 \mathrm{ml} / \mathrm{kg}$ peso corporeo $)$ allo scopo di indurre e mantenere una diuresi idrica massimale con osmolarità urinaria inferiore a 100 mOsml kg. Dopo un periodo di 90 minuti di stabilizzazione, fu raccolta l'urina per 30 minuti (minzioni spontanee). Durante questo periodo furono anche prelevate 3 provette di sangue a distanza di 7 minuti l'una dall'altra.

Nel primo protocollo, dopo il prelievo basale, fu iniziata l'infusione endovenosa di 2 ore di EAA (Fe 59092 Baxter, $5.4 \%$ ) alla velocità di $2.7 \mathrm{ml} / \mathrm{min} / 1.73 \mathrm{~m}^{2}$ superficie corporea, equivalente a $145 \mathrm{mg} / \mathrm{min} /$ $1.73 \mathrm{~m}^{2}(1,041 \mu \mathrm{mol})$; nel secondo protocollo la soluzione MIX-AA (Freamine III Baxter, 8.5\% diluita al $5.6 \%$ con acqua sterile) fu infusa per 2 ore alla stessa velocità di 2.7 $\mathrm{ml} / \mathrm{min} / 1.73 \mathrm{~m}^{2}$ pari a $145 \mathrm{mg} / \mathrm{min} /$ $1.73 \mathrm{~m}^{2}(1.235 \mu \mathrm{mol})$ suddivisi in 76 e $69 \mathrm{mg} / \mathrm{min} / 1.73 \mathrm{~m}^{2}$ di AA essenziali e non-essenziali rispettivamente $\left(549\right.$ e $\left.686 \mu \mathrm{mol} / \mathrm{min} / 1.73 \mathrm{~m}^{2}\right)$. Nell'Appendice viene elencata la composizione delle due soluzioni. Durante gli ultimi 20 minuti di ciascuna delle due ore, in entrambe le infusioni, furono raccolte l'urina e 2 provette di sangue. All'inizio e al termine del test furono misurati peso corporeo, pressione arteriosa sistolica e diastolica.

\section{Determinazione ed analisi statistica}

Abbiamo calcolato la clearance dell'Inutest e del PAH utilizzando i valori della loro concentrazione urinaria e la media dei livelli plasmatici rilevati durante i periodi di raccolta. I valori plasmatici di urea, insulina e glucagone furono misurati sull'ultima provetta raccolta in ogni periodo; l'urea urinaria fu valutata in ciascun periodo di raccolta delle urine. All'inizio e al termine dell'infusione di AA furono misurati in plasma ed urine gli elettroliti, gli AA e l'osmolarità. Tutte le determinazioni furono eseguite due volte. Il sangue per la determinazione di Inutest, PAH, sodio, osmolarità, urea e insulina fu raccolto in provette con litioesparina; quello per la misurazione degli AA in sodio-eparina; per il glucagone in EDTA + trasilolo. Inutest e PAH in plasma e urine furono determinati come descritto nello studio precedente (4). Gli AA furono misurati mediante cromatografia a scambio ionico; glucagone ed insulina con metodo radioimmunologico (Sorin Biomedica, Saluggia, Italia; e RIA-Mat, Byk Gulden, Italia rispettivamente); i valori di urea plasmatica ed urinaria mediante procedure automatizzate e standardizzate di laboratorio usando una macchina Hitachi 737. Gli elettroliti plasmatici ed urinari furono analizzati con fotometria a fiamma. L'osmolarità plasmatica ed urinaria fu misurata usando un osmometro Fiske (tecnica del punto di congelamento). Le clearance renali furono calcolate con la formula standard.

Di seguito vengono definiti alcuni parametri:

- Frazione di filtrazione $(\mathrm{FF})=$ FG/FPR;

- Frazione escreta del sodio ( $\mathrm{FeNa}$ $\%)=(\mathrm{Na}$ urinario/Na plasmatico $) \mathrm{x}$ (Inutest plasmatico/Inutest urinario) x 100 ;

- Carico filtrato di $\mathrm{AA}=$ (concentrazione plasmatica AA x FG);

- Riassorbimento assoluto di AA = (carico filtrato AA) - (escrezione urinaria di $\mathrm{AA}$ );

- Frazione di riassorbimento AA $(\%)=($ riassorbimento assoluto AA) x $100 /($ carico filtrato di AA);
- Frazione escreta di AA (FeAA \%) $=(100$ - frazione riassorbita AA $)$;

- Carico filtrato di urea = (urea plasmatica x FG);

- Frazione escreta di urea (FeUrea $\%)=($ Urea urinaria/Urea plasmatica) $x$ (Inutest plasmatico/Inutest urinario) x 100;

- Clearance dell'acqua libera $\left(\mathrm{CH}_{2} \mathrm{O}\right)$ $=($ flusso urinario $)-($ clearance osmolare); poi viene correlata a 100 $\mathrm{ml}$ di filtrato glomerulare (FG).

I valori furono espressi come media all'interno dei gruppi \pm deviazioni standard (DS). Per le variabili di cui erano disponibili tre valori fu eseguita l'analisi della varianza per blocchi randomizzati. Le differenze statistiche all'interno dei gruppi furono valutate usando il test di Duncan. Nei casi in cui erano disponibili solo due valori fu utilizzato il test t-Student per dati appaiati. La correlazione tra variabili fu eseguita usando un'analisi della regressione lineare.

\section{Risultati}

Nessuna delle due soluzioni di AA ha modificato il peso corporeo (68.2 \pm 6.5 vs. $69.5 \pm 6.1 \mathrm{~kg}$ con EAA e $69.8 \pm 6.3$ vs. $70.1 \pm 6.4 \mathrm{~kg}$ con MIX-AA), la pressione arteriosa sistolica $(123 \pm 5$ vs. $125 \pm 7 \mathrm{mmHg}$ con $\mathrm{EAA}$ e $122 \pm 5$ vs. $127 \pm 6$ $\mathrm{mmHg}$ con MIX-AA) o diastolica ( $83 \pm 6$ vs. $86 \pm 10 \mathrm{mmHg}$ con EAA e $80 \pm 2$ vs. $85 \pm 5 \mathrm{mmHg}$ con MIXAA), e l'ematocrito $(44.0 \pm 1$ vs. $43.5 \pm 1 \%$ con EAA e $43.8 \pm 2$ vs. $42.9 \pm 2 \%$ con MIX-AA).

\section{Amino acidi}

L'infusione di entrambe le soluzioni ha provocato un aumento considerevole dei livelli totali di AA (Tab. I). Naturalmente quando è stata somministrata EAA hanno contribuito a tale incremento solo gli AA essenziali, mentre con l'infusione di MIX-AA erano implicati entrambi i tipi di AA. Il riassorbimento assoluto di AA è aumentato significativamente in entrambi i protocolli, mentre la frazione riassorbita è rimasta invariata durante l'infusione di EAA ed è dimi- 
TABELLA I - CONCENTRAZIONE PLASMATICA E TRASPORTO TUBULARE DEGLI AA TOTALI, ESSENZIALI E NON-ESSENZIALI PRE E POST INFUSIONE DI EAA E MIX-AA

\begin{tabular}{|c|c|c|c|c|}
\hline & \multicolumn{2}{|c|}{ EAA } & \multicolumn{2}{|c|}{ MIX-AA } \\
\hline & Pre & Post & Pre & Post \\
\hline \multicolumn{5}{|l|}{ AA plasmatici $(\mu \mathrm{mol} / 100 \mathrm{ml})$} \\
\hline Totali & $269 \pm 34$ & $462 \pm 53^{*}$ & $272 \pm 39$ & $502 \pm 84 *$ \\
\hline Essenziali & $97 \pm 16$ & $284 \pm 48 *$ & $87 \pm 13$ & $180 \pm 33^{*}$ \\
\hline Non-essenziali & $172 \pm 22$ & $178 \pm 17$ & $185 \pm 35$ & $322 \pm 56^{*}$ \\
\hline \multicolumn{5}{|l|}{ Riassorbimento assoluto di AA $\left(\mu \mathrm{mol} / \mathrm{min} / 1.73 \mathrm{~m}^{2}\right)$} \\
\hline Totali & $264 \pm 34$ & $473 \pm 57 *$ & $268 \pm 47$ & $586 \pm 90 *$ \\
\hline Essenziali & $94 \pm 14$ & $291 \pm 43^{*}$ & $84 \pm 17$ & $245 \pm 37 *$ \\
\hline Non-essenziali & $170 \pm 20$ & $182 \pm 19$ & $184 \pm 29$ & $341 \pm 58 *$ \\
\hline \multicolumn{5}{|l|}{ Frazione riassorbita di AA (\%) } \\
\hline Totali & $98.3 \pm 0.2$ & $98.5 \pm .04$ & $98.6 \pm 03$ & $95.7 \pm 13^{*}$ \\
\hline Essenziali & $98.9 \pm 0.3$ & $98.6 \pm 0.6$ & $99.0 \pm 0.4$ & $98.3 \pm 0.7$ \\
\hline Non-essenziali & $98.1 \pm 0.4$ & $98.3 \pm 0.4$ & $98.6 \pm 03$ & $94.4 \pm 02 *$ \\
\hline
\end{tabular}

$* p<0.01$ vs Pre

nuita con quella di MIX-AA. La frazione escreta di AA è aumentata significativamente (da $1.5 \pm 0.4$ a $4.7 \pm$ $1.6 \% ; \mathrm{p}<0.05$ ) durante l'infusione di MIX-AA.

\section{Emodinamica renale}

La Figura 1 mostra come durante l'infusione di EAA non si è osservato un aumento significativo del FG; il valore medio basale è stato di $98 \pm 6$ $\mathrm{ml} / \mathrm{min} / 1.73 \mathrm{~m}^{2}$ rispetto ai $103 \pm 23 \mathrm{e}$ $104 \pm 22$ dopo 1 e 2 ore di infusione rispettivamente. Con la somministrazione di MIX-AA, il FG era $104 \pm 6$ alla determinazione basale, $109 \pm 15$ dopo 1 ora ed è aumentato significativamente a $122 \pm 13 \mathrm{ml} / \mathrm{min} / 1.73$ $\mathrm{m}^{2}$ dopo 2 ore di infusione $(\mathrm{p}<0.05)$. Un andamento analogo si è osservato per il FPR: non ci sono state variazioni durante l'infusione di EAA $\left(485 \pm 31 \mathrm{ml} / \mathrm{min} / 1.73 \mathrm{~m}^{2}\right.$ basale, $486 \pm 53$ dopo 1 ora e $485 \pm 42$ dopo 2 ore); con MIX-AA il FPR medio basale è stato $488 \pm 46,489 \pm 85$ dopo 1 ora e quindi è aumentato significativamente a $572 \pm 34 \mathrm{ml} / \mathrm{min} /$ $1,73 \mathrm{~m}^{2}$ dopo 2 ore $(\mathrm{p}<0.05)$. La frazione di filtrazione non è variata durante entrambe le infusioni (EAA: 21 $\pm 3 \%$ basale, $22 \pm 3$ ad 1 ora e $22 \pm 2$ a 2 ore vs. $22 \pm 2 \%, 23 \pm 2$ e $23 \pm 4$ con MIX-AA).

\section{Metabolismo azotato}

La concentrazione plasmatica media di ornitina si è ridotta significativamente dopo 2 ore di infusione di EAA (da $86 \pm 25$ a $60 \pm 15, \mu \mathrm{mol} / \mathrm{l}$; $\mathrm{p}<0.01$ ), mentre è aumentata significativamente dopo la somministrazione per lo stesso tempo di MIX-AA (da $96 \pm 28$ a $139 \pm 32 \mu \mathrm{mol} / 1$; $\mathrm{p}<0.01)$. Il valore medio plasmatico di arginina si è innalzato dopo MIXAA $($ da $60 \pm 22$ a $185 \pm 29 \mu \mathrm{mol} / \mathrm{l}$; $\mathrm{p}<0.01$ ), ma anche dopo EAA (da 57 \pm 14 a $99 \pm 38, \mu \mathrm{mol} / \mathrm{l} ; \mathrm{p}<0.05$ ). L'urea plasmatica è rimasta invariata durante infusione di EAA mentre è salita (sebbene non in maniera statisticamente significativa) con MIX-AA (Tab. II). Né il carico filtrato di urea né l'escrezione urinaria di urea sono cambiati in risposta a EAA, mentre entrambi sono aumentati evidentemente con l'infusione di MIX-AA (Tab. III); inoltre l'incremento dell'escrezione urinaria di urea dopo 2 ore di infusione di MIX-AA è rimasto significativo $(\mathrm{p}<0.05)$ anche dopo la normalizzazione del FG (basale $21 \pm$ $2.1 \mathrm{mg} / \mathrm{dl} \mathrm{FG}$; a 2 ore $26 \pm 4.3 \mathrm{mg} / \mathrm{dl}$ FG). La frazione escreta di urea è alta con la somministrazione di MIX$\mathrm{AA}$, sebbene la variazione non abbia raggiunto livelli statisticamente significativi (Tab. II). È stata rilevata

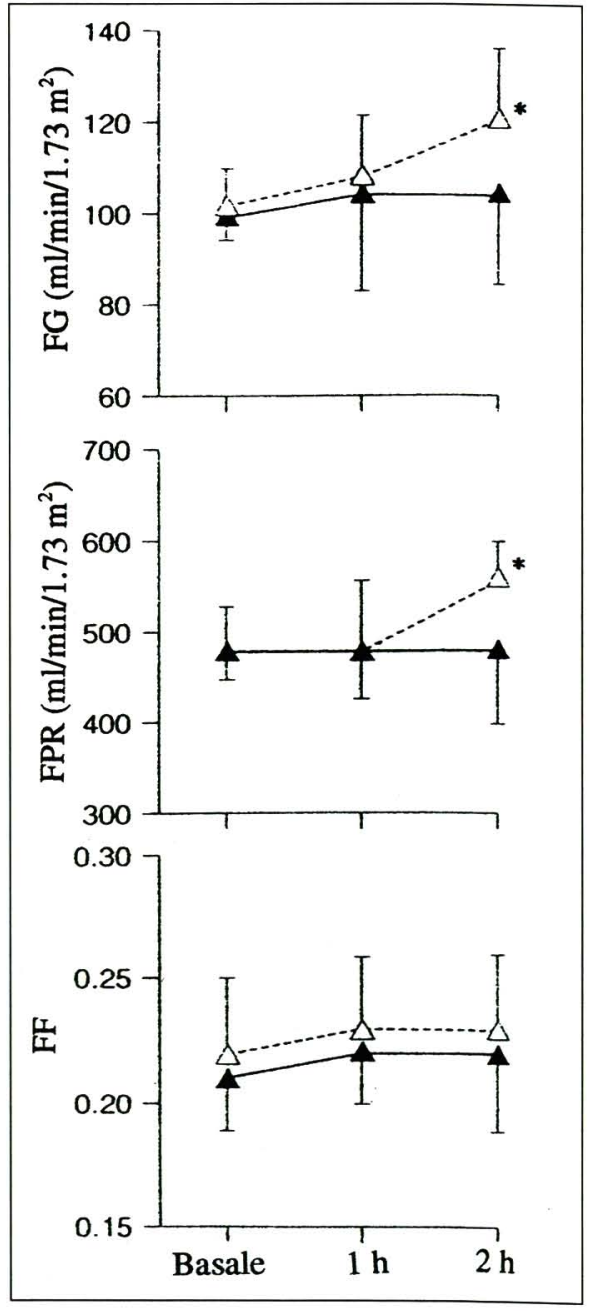

Fig. 1 - Variazioni dell'emodinamica renale indotte dalla infusione di EAA $(\boldsymbol{\Lambda}-\boldsymbol{\Lambda})$ e MIX-AA $(\Delta----\Delta) .(p<0.05$ vs basale $)$ 
TABELLA II - METABOLISMO AZOTATO DURANTE INFUSIONE DI EAA E MIX-AA

\begin{tabular}{lrrrrrr}
\hline & \multicolumn{3}{c}{ EAA } & \multicolumn{3}{c}{ MIX-AA } \\
\cline { 2 - 7 } & Basale & $1 \mathrm{~h}$ & $2 \mathrm{~h}$ & Basale & $1 \mathrm{~h}$ & $2 \mathrm{~h}$ \\
\hline Urea plasmaitca $(\mathrm{mg} / \mathrm{dl})$ & $32.3 \pm 1.2$ & $31.6 \pm 1.4$ & $32.0 \pm 2.3$ & $28.5 \pm 1.2$ & $32.6 \pm 1.6$ & $34.7 \pm 2.2$ \\
Carico filtrato di urea $\left(\mathrm{mg} / \mathrm{min} / 1.73 \mathrm{~m}^{2}\right)$ & $31.7 \pm 3.1$ & $33.7 \pm 4.2$ & $32.8 \pm 5.7$ & $32.4 \pm 3.8$ & $35.3 \pm 4.5$ & $40.4 \pm 7.3^{*}$ \\
Escrezione urinaria di urea $\left(\mathrm{mg} / \mathrm{min} / 1.73 \mathrm{~m}^{2}\right)$ & $23.6 \pm 6.7$ & $22.2 \pm 8.1$ & $23.4 \pm 5.3$ & $20.7 \pm 2.1$ & $22.6 \pm 2.3$ & $30.6 \pm 7.5^{*}$ \\
Frazione escreta di urea $(\%)$ & $68 \pm 17$ & $69 \pm 14$ & $71 \pm 18$ & $63 \pm 10$ & $65 \pm 17$ & $77 \pm 22$ \\
\hline
\end{tabular}

$* p<0.05$ vs basale

TABELLA III - VALORI PLASMATICI DI INSULINA, GLUCAGONE E RAPPORTO GLUCAGONE/INSULINA DURANTE L'INFUSIONE DI EAA E MIX-AA

\begin{tabular}{|c|c|c|c|c|c|c|}
\hline & \multicolumn{3}{|c|}{ EAA } & \multicolumn{3}{|c|}{ MIX-AA } \\
\hline & Basale & $1 \mathrm{~h}$ & $2 \mathrm{~h}$ & Basale & $1 \mathrm{~h}$ & $2 \mathrm{~h}$ \\
\hline Insulina plasmatica $(\mathrm{ml} / \mathrm{U} / \mathrm{I})$ & $6.3 \pm 13$ & $12.1 \pm 3.0^{*}$ & $11.6 \pm 3.5^{*}$ & $6.5 \pm 3.2$ & $13.1 \pm 4.0^{*}$ & $10.2 \pm 6.6^{*}$ \\
\hline Glucagone plasmatico $(\mathrm{pg} / \mathrm{ml})$ & $120 \pm 67$ & $158 \pm 59$ & $177 \pm 80 *$ & $118 \pm 36$ & $167 \pm 31 *$ & $243 \pm 45^{*}$ \\
\hline Glucagone/Insulina plasmatica & $19 \pm 6$ & $13 \pm 5^{*}$ & $15 \pm 8$ & $18 \pm 7$ & $13 \pm 2 *$ & $24 \pm 54 * \S$ \\
\hline
\end{tabular}

$* p<0.05$ vs basale; $\$ p<0.05$ vs $1 \mathrm{~h}$

TABELLA IV - TRASPORTO DI Na E ACQUA DOPO INFUSIONE DI EAA E MIX-AA

\begin{tabular}{lcccc}
\hline & \multicolumn{2}{c}{ EAA } & \multicolumn{2}{c}{ MIX-AA } \\
\cline { 2 - 5 } & \multicolumn{1}{c}{ Pre } & Post & Pre & Post \\
\hline Na plasmatico $(\mathrm{mmol} / \mathrm{L})$ & $141 \pm 0.8$ & $140 \pm 1.0$ & $140 \pm 0.6$ & $140 \pm 0.9$ \\
$\mathrm{FeNa}(\%)$ & $1.2 \pm 0.6$ & $13 \pm 0.7$ & $1.3 \pm 0.6$ & $2.0 \pm 0.8^{*}$ \\
Osmolarità plasmatica $\left(\mathrm{mOsm} / \mathrm{kg} \mathrm{H} \mathrm{H}_{2} \mathrm{O}\right)$ & $285 \pm 3.4$ & $286 \pm 1.6$ & $286 \pm 4.1$ & $287 \pm 3.4$ \\
Osmolarità urinaria $\left(\mathrm{mOsm} / \mathrm{kg} \mathrm{H}_{2} \mathrm{O}\right)$ & $89 \pm 15$ & $92 \pm 11$ & $95 \pm 13$ & $93 \pm 9$ \\
Flusso urinario $\left(\mathrm{ml} / \mathrm{min} / / 1.73 \mathrm{~m}^{2}\right)$ & $11.6 \pm 0.7$ & $9.8 \pm 4.0$ & $11.1 \pm 2.2$ & $143 \pm 1.8^{*}$ \\
Clearance dell'acqua libera $\left(\mathrm{CH}_{2} \mathrm{O} / 100 \mathrm{ml} \mathrm{GF}\right)$ & $7.8 \pm 04$ & $7.6 \pm 2.5$ & $6.6 \pm 1.2$ & $9.8 \pm 0.9$ \\
\hline
\end{tabular}

$* p<0.05$ vs Pre

una correlazione significativa tra $\mathrm{i}$ valori individuali di FG e di escrezione urinaria di urea $(r=0.66 ; p<0.05)$ durante l'infusione di MIX-AA.

\section{Ormoni}

I livelli plasmatici di glucagone ed insulina sono aumentati significativamente durante l'infusione di entrambe le soluzioni; con EAA il rapporto glucagone/insulina è diminuito significativamente dopo 1 ora ed è quindi tornato al valore basale dopo 2 ore, mentre con l'infusione di MIX-AA, dopo la riduzione osservata ad 1 ora, dopo 2 ore si è assistito ad un rialzo significativo rispetto ai valori basali (Tab. III). Le variazioni di FG sono state correlate con i livelli plasmatici di glucagone e con il rapporto plasmatico glucagone/insulina (Fig. 3). La correlazione era significativa $(\mathrm{r}=0.40$; $\mathrm{p}<0.05$ ) solo con quest'ultima infusione.

\section{Acqua e sodio}

Durante entrambe le infusioni non sono cambiate né la concentrazione plasmatica media di sodio, né l'osmolarità plasmatica (Tab. IV). L'osmolarità urinaria è rimasta al di sotto di $100 \mathrm{mOsm} / \mathrm{kg} \mathrm{H}_{2} \mathrm{O}$ durante entrambi i test. Il flusso urinario e la clearance dell' acqua libera sono rimasti stabili durante la somministrazione di EAA, mentre sono aumentati con l'infusione di MIX-AA. La Fe- 
$\mathrm{Na}$ non è stata modificata da EAA ma è stata incrementata da MIX-AA (Tab. IV).

\section{Discussione}

Poiché l'urea rappresenta il principale prodotto finale del catabolismo delle proteine, la variazione della sua escrezione urinaria, associata ai livelli plasmatici di glucagone ed insulina, rappresentano degli indicatori affidabili degli eventi metabolici splancnici che fanno seguito all'introduzione di proteine o AA. Il presente studio ha dimostrato che $i$ cambiamenti di tali indicatori del metabolismo azotato, ottenuti durante l'infusione di due differenti soluzioni di AA, sono correlati temporalmente e quantitativamente alle modificazioni dell'emodinamica renale che conseguono alla somministrazione di AA.

Durante la somministrazione di MIX-AA, che conteneva AA elettivamente metabolizzati a livello splancnico, si è verificato un incremento dell'escrezione urinaria di urea, del carico filtrato di urea e del rapporto plasmatico glucagone/insulina (che è noto essere il vero determinante della gluconeogenesi e ureagenesi $(13,14)$ dopo 2 ore di infusione, in corrispondenza dell'aumento del FG e del FPR. Il fatto che l'incremento dell'escrezione di urea sia rimasto significativo anche dopo la normalizzazione del FG e che tale aumento si sia verificato senza una contemporanea riduzione dei livelli plasmatici di urea, dimostra chiaramente che è aumentata la produzione di urea e, pertanto, che è stato stimolato il metabolismo epatico. Un'ulteriore osservazione a riprova che l'ureagenesi fosse aumentata durante l'infusione di MIX-AA è che l'escrezione di urea sia stata più consistente rispetto a quanto ci si sarebbe aspettato dalla separazione equimolare dell'arginina infusa in urea ed ornitina (un singolo passaggio enzimatico, catalizzato dall' arginasi (15).

Al contrario l'infusione di EAA non ha provocato alcun cambiamento dell'emodinamica renale nonostante sia-

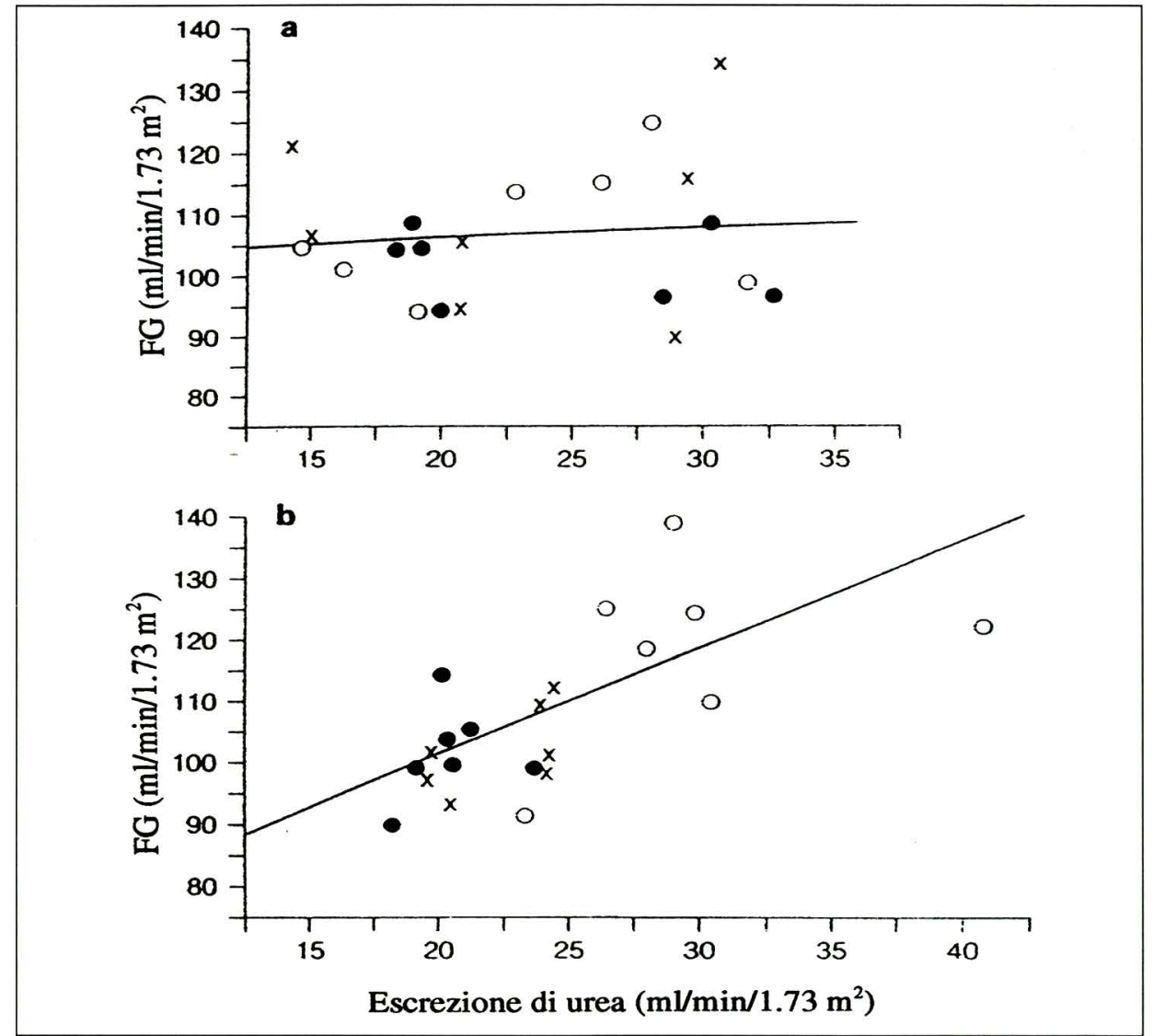

Fig. 2 - Relazione tra il FG e l'escrezione urinaria di urea prima $(\bullet), 1(x)$ e $2(O)$ ore dopo l'inizio dell'infusione di EAA (a) $(r=0.01 ; p=0.9)$ e MIX-AA (b) $(r=0.66 ; p<0.05)$
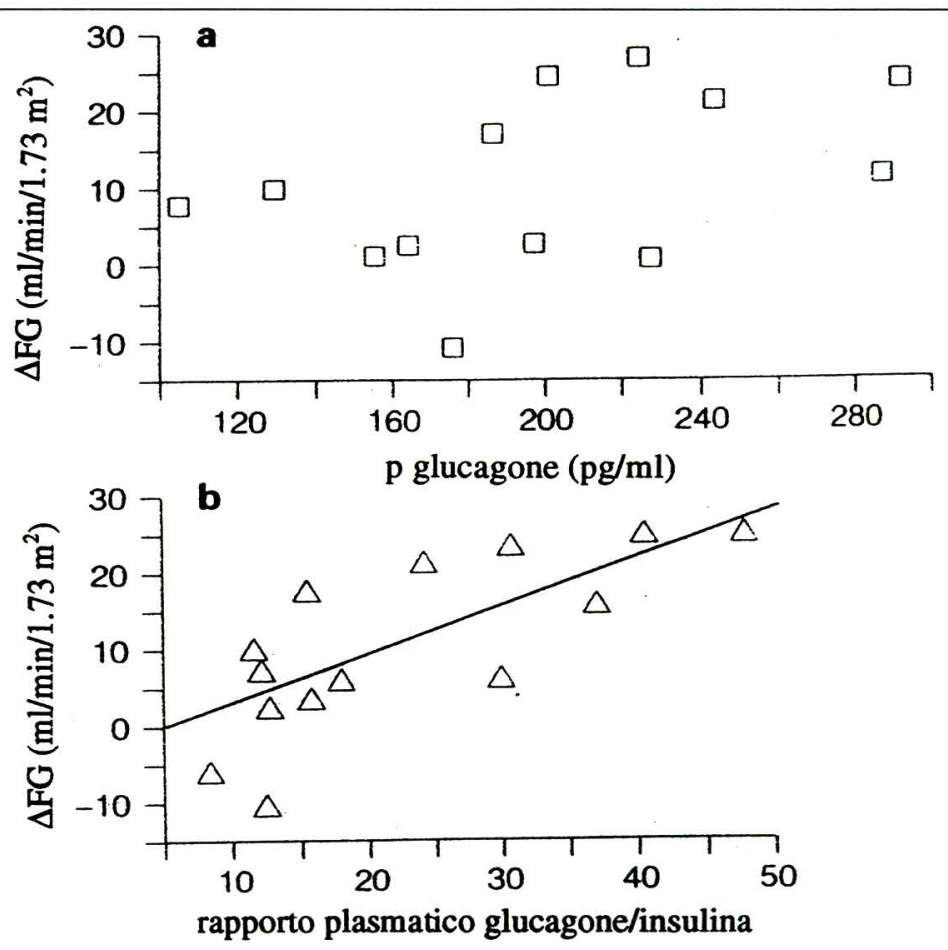

Fig. 3 - Correlazione tra la variazione di $F G(\triangle F G)$, glucagone plasmatico (a) e rapporto plasmatico glucagonefinsulina $(b)(r=0.40 ; p<0.05)$ durante l'infusione di $M I X-A A$. 
no stati raggiunti al termine del test livelli plasmatici di AA equivalenti a quelli ottenuti con MIX-AA. È noto che gli AA essenziali non sottostiano al metabolismo splancnico (16) o all'ossidazione e vengano utilizzati prevalentemente per la sintesi proteica o (nel caso dei tre AA a catena ramificata) metabolizzati a livello muscolare sotto il controllo dell'insulina (17). Non sono state osservate variazioni dell'escrezione di urea, del carico filtrato di urea o del rapporto plasmatico glucagone/insulina ed inoltre, il decremento dei livelli plasmatici di ornitina che si è osservato (e il corrispondente aumento dei valori plasmatici di arginina) in seguito all'infusione di EAA può essere interpretato come indicatore della ridotta ureagenesi. I nostri risultati sono in accordo con la dimostrazione che l'FG non aumenta dopo somministrazione di D-serina e cistina (2) o di AA a catena ramificata (3-5), nessuno dei quali viene metabolizzato a livello splancnico, mentre è incrementato da una miscela di AA gluconeogenetici (3).

La contemporanea stimolazione del metabolismo epatico e della funzione renale da noi osservata fa ipotizzare che questi due eventi possano essere in rapporto di causa-effetto.

Nonostante Baines et al (18) e El Sayed et al (19) abbiano dimostrato che alcuni AA in vitro provocavano direttamente iperfiltrazione e vasodilatazione renale, Premen (2) ha dimostrato che l'infusione diretta nell'arteria renale di cani di tre AA non essenziali (serina, alanina e prolina) non modificavano l'emodinamica renale, mentre 1'FG aumentava dopo l'infusione sistemica degli stessi. Ciò suggerisce che per indurre iperfiltrazione renale in vivo sia necessario un passaggio metabolico intermedio. Gli AA non-essenziali sono considerati potenti stimolatori della secrezione di glucagone (13) e una considerevole mole di lavori ha dimostrato l'implicazione del glucagone nell'induzione di iperfiltrazione renale da parte degli AA. Se la secrezione endogena di glucagone viene inibita dalla somministrazione di somatostatina $(6,8)$ o bloccata dalla pancreatecto- mia (7-9) non si osserva vasodilatazione renale dopo infusione di AA; in tali condizioni sperimentali, le modificazioni dell'emodinamica renale vengono ripristinate solamente mediante somministrazione di glucagone $(6,8,9)$. Comunque è stato dimostrato che l'infusione di una quantità di glucagone, sufficiente a raggiungere nel sangue periferico una concentrazione post-prandiale fisiologica, non provoca iperfiltrazione, e che si ottiene una risposta renale solo quando si raggiungono livelli sovrafisiologici (sebbene probabilmente essi siano fisiologici a livello epatico) (10) oppure quando il glucagone viene somministrato in associazione ad urea e cAMP (11). Inoltre, Premen (20) ha dimostrato che il glucagone provoca i suoi effetti sull'emodinamica renale solo se infuso nella vena porta, giungendo alla conclusione che esso induca il rilascio da parte del fegato di una sostanza con effetto vasodilatatore sul rene. Nel nostro studio l'aumento del FG provocato dagli AA è avvenuto contemporaneamente al più alto incremento dei livelli di glucagone plasmatico rispetto alla valutazione basale; comunque, la percentuale di incremento non era superiore all' $80 \%$ e i livelli plasmatici erano al di sotto delle concentrazioni sovrafisiologiche necessarie ad incrementare il FG. Oltretutto non abbiamo trovato correlazioni significative tra i livelli plasmatici di glucagone e le variazioni del FG durante l'infusione di MIX-AA. Tutto ciò fa supporre che il glucagone, piuttosto che essere un mediatore indipendente, sia parte di una cascata di eventi che portano alla stimolazione dell'emodinamica renale.

L'infusione di MIX-AA provoca un aumento del rapporto plasmatico glucagone/insulina che viene considerato il vero attivatore di una serie di eventi metabolici splancnici tra cui la gluconeogenesi e l'ureagenesi (13, 14). Abbiamo trovato una correlazione temporale significativa sia tra l'FG e rapporto plasmatico glucagone/insulina, sia tra FG ed escrezione urinaria di urea. Sebbene queste correlazioni di per sé non implichino una relazione causale, dimostrano che l'attivazione splancnica è associata all'iperfiltrazione renale indotta da AA. Ahloulay et al $(10,11)$ hanno proposto che l'urea e il cAMP possano rappresentare il legame tra il metabolismo splancnico degli AA e il meccanismo intrarenale responsabile dell'iperfiltrazione.

Nel modello teorico proposto recentemente da Woods (1), tale meccanismo, in accordo con la maggior parte (ma non tutti) (21) dei dati di letteratura, è stato identificato con il feedback tubulo glomerulare (FTG). Variazioni del riassorbimento di sodio in prossimità dell'apparato iuxtaglomerulare provocano una modulazione dell' attività del FTG $(1,12,22$ 24) e del FG. Nel nostro studio, le due infusioni di AA hanno avuto effetti diversi sulla funzione tubulare: con la somministrazione di MIX-AA abbiamo osservato un aumento della frazione escreta degli AA (dovuta ad un differente trasporto degli AA essenziali e non-essenziali, che probabilmente è spiegato dalle loro proprietà strutturali) (25), della frazione escreta del sodio e della clearance dell'acqua libera. Supponendo che i test siano stati condotti in condizioni di diuresi idrica, ciò significa che si è verificato un aumento del riassorbimento dei soluti nel segmento diluente (26). Questo risultato è in accordo con quelli di altri studi su animali che hanno mostrato un incremento del riassorbimento di sodio a livello della porzione spessa della branca ascendente dell'ansa di Henle in seguito a carico di proteine (27, $28)$. E stato ipotizzato che il glucagone $(29,30)$, il cAMP epatico $(31)$ e l'urea (32) possano modificare la concentrazione di sodio cloruro nel segmento diluente e nel fluido che raggiunge la macula densa e in tal modo variare l'attività del FTG. Questi risultati spingono ad eseguire ulteriori studi sulla relazione tra stimolazione splancnica e tubulare in corso di infusione di AA non-essenziali.

In conclusione, questo studio sostiene l'ipotesi che il metabolismo splancnico degli AA (l'equilibrio tra secrezione di glucagone e insulina, gluconeogenesi e ureagenesi) costituisca un passaggio fondamentale nel 
processo che porta all'iperfiltrazione renale indotta da AA; rimane ancora da stabilire come esso possa influenzare il meccanismo renale finale.

\section{Ringraziamenti}

Gli Autori ringraziano la Dr.ssa Lise Bankir (Hôpital Necker, Parigi) per il supporto e i preziosi consigli.

Questo studio è stato finanziato dall' "Associazione per il Bambino Nefropatico".

Alcuni dati contenuti in questo articolo sono stati presentati al $26^{\circ}$ Meeting dell'American Society of Nephrology, Boston, Mass., Novembre 1993. ("Effects on renal hemodynamics of different IV amino acid solutions" [abstract] Am Soc Nephrol 1993; 4: 576).

\begin{tabular}{lcc} 
APPENDICE - COMPOSIZIONE \\
DELLE DUE SOLUZIONI DI AMI- \\
NO ACIDI (g/l) \\
\hline & EAA & MIX-AA \\
\hline & 6.5 & 3.7 \\
\hline Valina & 8.8 & 5.1 \\
Leucina & 5.6 & 3.9 \\
Isoleucina & 2.0 & 0.85 \\
Triptofano & 4.0 & 2.2 \\
Treonina & 8.8 & 3.16 \\
Fenilalanina & 8.8 & 2.9 \\
Metionina & 9.0 & 5.7 \\
Lisina & 2.5 & 1.6 \\
Istidina & 0.3 & 0.13 \\
Cisteina & - & 5.3 \\
Arginina & - & 3.9 \\
Alanina & - & 7.8 \\
Glicina & - & 3.3 \\
Serina & - & 6.2 \\
Prolina & & \\
& 56.3 & 55.74 \\
Totale & & \\
\hline &
\end{tabular}

\section{BIBLIOGRAFIA}

1. Woods LL. Mechanisms of renal hemodynamic regulation in response to protein feeding. Kidney Int 1993; 44: 659-75.

2. Premen AJ. Nature of the renal hemodynamic action of amino acids in dogs. Am J Physiol 1989; 256: F516-23.

3. Castellino P, Levin R, Shohat $\mathbf{J}$, and De Fronzo R. Effect of specific amino acid groups on renal hemodynamics in humans. Am J Physiol 1990; 258: F992-7.

4. Claris-Appiani A, Assael BM, Tirelli S, Marra G, Cavanna G. Lack of glomerular hemodynamic stimulation after infusion of branchedchain amino acids. Kidney Int 1988; 33: $91-4$

5. Lee KE, Summerill RA. Glomerular filtration rate following administration of individual amino acids in conscious dogs. Q J Exp Physiol 1982; 67: 459-65.

6. Castellino P, Giordano C, Perna A, De Fronzo RA. Effects of plasma amino acid and hormone levels on renal hemodynamics in humans. Am J Physiol 1988; 255: F444-9.

7. De Santo NG, Coppola S, Anastasio P, et al. Pancreatectomy abolishes the renal hemodynamic response to a meat meal in man. Nephron 1990; 55: 85-6.

8. Friedlander G, Blanchet-Benque F, Nitemberg A, Laborie C, Assan R, Amiel C. Glucagon secretion is essential for amino acid-induced hyperfiltration in man. Nephrol Dial Transplant 1990; 5: 110-7.

9. Premen AJ, Powell DA, Carrol RG, Dobbins DE. Renal vascular response to amino acids: effect of pancreatectomy. Am J Physiol 1990; 258: F1154-63.

10. Ahloulay M, Bouby N, Machet F, Kubrusly M, Coutaud C, Bankir L. Effects of glucagon on glomeru- lar filtration rate and urea and water excretion. Am J Physiol 1992; 263: F24-36.

11. AhloulayM, Hassler C, Bankir L. Glucagon-induced elevation in GFR: Role of liver-borne cAMP and urea. Kidney Int 1995; 47: 685 (abstr).

12. Woods LL, Mizelle HL, Montani JP, Hall JE. Mechanisms controlling renal hemodynamics and electrolyte excretion during amino acids. Am J Physiol 1986; 251: F303-12.

13. Unger RH, Orci L. Glucagon and the A-cell: physiology and pathophysiology. N Engl J Med 1981; 304: 1518-75.

14. Parilla R, Goodman MN, Toews CJ. Effect of glucagon: insulin ratios on hepatic metabolism. Diabetes 1974; 23: 725-31.

15. Meijer AJ, Lamers WH, Chamuleau RAF. Nitrogen metabolism and ornithine cycle function. Physiol Rev 1990; 70: 701-48.

16. De Ferrari G, Garibotto G, Robaudo C, Sala M, Tizianello A Splanchnic exchange of amino acids after amino acid ingestion in patients with chronic renal insufficiency. Am J Clin Nutr 1988; 48: 72-83.

17. Fukagawa NK, Minaker KL, Young VR, Rowe JW. Insulin dosedependent reduction in plasma amino acids in man. Am J Physiol 1986; 250: E13-7.

18. Baines AD, Ho P, James H Metabolic control of renal vascular resistance and glomerulotubular balance. Kidney Int 1985; 27: 848-54.

19. El Sayed AA, Haylor J, El Nahas AM. Mediators of the direct effects of amino acids on the rat kidney. Clin Sci 1991; 81: 427-32.

20. Premen AJ. Potential mechanisms mediating postprandial renal hyperemia and hyperfiltration. FASEB J 1988; 2: 131-7.

21. Brown SA, Navar LG. Singlenephron responses to systemic ad- 
ministration of amino acids in dogs. Am J Physiol 1990; 259: F739-46.

22.

Claris-Appiani A, Assael BM, Tirelli S, Cavanna G, Corbetta C, Marra G. Proximal tubular function and hyperfiltration during amino acid infusion in man. Am J Nephrol 1988; 8: 96-101.

23. Woods LL, Young EW. Impaired renal hemodynamic response to protein feeding in dogs with experimental Fanconi syndrome. Am J Physiol 1991; 261: F14-21.

24. Woods LL, Smith BE, De Young DR. Regulation of renal hemodynamics after protein feeding: Effects of proximal and distal diuretics. Am J Physiol 1993; 264: R33744.

25. Silbernagl S. The renal handling of amino acids and oligopeptides. Physiol Rev 1988; 68: 911 1007.

26. Danovitch GM, Bricker NS. Influence of volume expansion on $\mathrm{NaCl}$ reabsorption in the diluting segments of the nephron: a study using clearance methods. Kidney Int 1976; 10: 229-38.

27. Bouby N, Bankir L. Effect of high protein intake on sodium, potassium-dependent adenosine triphosphatase activity in the thick ascending limb of Henle's loop in the rat. Clin Sci 1988; 74: 319-29.

28. Seney FD Jr, Persson AEG, Wright FS. Modification of tubuloglomerular feedback signal by dietary protein. Am J Physiol 1987; 252: F83-90.

29. Bailly C, Roinel N, Amiel C. PTH-like glucagon stimulation of $\mathrm{Ca}$ and $\mathrm{Mg}$ reabsorption in Henle's loop of the rat. Am J Physiol 1984; 246: F205-12.

30. Di Stefano A, Wittner M, Nitschke R, et al. Effects of glucagon on $\mathrm{Na}^{+}, \mathrm{Cl}^{-}, \mathrm{K}^{+}, \mathrm{Mg}^{2+}$ and $\mathrm{Ca}^{2+}$ transport in cortical and medullary thick ascending limbs of mouse kidney. Pflugger Arch 1989; 414: 640-6.

31. Bankir L, Kriz W. Adaptation of the kidney to protein intake and to urine concentrating activity: similar consequences in health and CRF. Kidney Int 1995; 47: 7-24.

32. Bankir L, Ahloulay M, Bouby $\mathrm{N}$, et al. Is the process of urinary urea concentration responsible for a high glomerular filtration rate? J Am Soc Nephrol 1993; 4: 1091-103. 